\title{
Diretrizes para a Projetação do Ambiente Virtual de Aprendizagem - Flow
}

\author{
Anaís Schüler Bertoni \\ Mestre em Design \\ anais@d750.com.br \\ Sidnei Renato Silveira \\ Professor Titular da Faculdade de Informática do UniRitter \\ sidnei@uniritter.edu.br
}

\begin{abstract}
Resumo: Um sistema de projetação, para o Desenho Industrial, pode ser considerado como uma técnica de resolução de problemas, conhecida como metodologia, que tem o propósito de guiar a concepção de determinado produto. Uma infinidade de autores dessa área já desenvolveram e categorizaram metodologias projetuais. Porém, apenas alguns deles criaram métodos para projetação de produtos dígito-virtuais. Neste sentido, o presente trabalho tem como objetivo relatar, por meio de uma revisão bibliográfica, como o Projeto $\mathrm{E}$ guiou o desenvolvimento do AVA Flow e lhe proporcionou o alcance dos princípios de usabilidade, verificados através de uma avaliação heurística, trabalhados juntamente com princípios universais de projeto da área do Desenho Industrial.
\end{abstract}

Palavras-chave: usabilidade, Projeto E, interfaces gráficas com o usuário, ambientes virtuais de aprendizagem, avaliação heurística.

\section{Guidelines for Design of Flow Virtual Learning Environment}

Abstract: A projecting system, for design, can be regarded as a technique for solving problems, as known methodology, which is intended to guide the design of a product. A multitude of authors in this area has developed and categorized projective methodologies. However, only some of them have established methods for projecting virtual-digit products. This paper aims to present, through a literature review, how the E Project guided the development of virtual learning environment Flow and gave him the scope of usability principles, verified by a heuristic evaluation, worked together with the universal principles of Design.

Key Words: usability, E Project, graphical user interface, virtual learning environment and heuristic evaluation.

\section{Introdução}

$\mathrm{O}$ advento do computador foi um marco importante para o desenvolvimento da mídia digital. Lévy (1993, p.101) afirma que "foi esta inovação imprevisível que transformou a informática em um meio de massa para a criação, comunicação e simulação", por meio da Internet. Porém, conforme exemplifica Arantes (2005, p.64), a interação que se dá entre o homem e a máquina só é efetivada quando mediada por um dispositivo conhecido como interface, que "permite que a ação do homem, desde a mais simples, como apertar o teclado, seja reconhecida, processada pela máquina e devolvida para o usuário". 
As constantes mudanças no meio digital, conforme Kalbach (2009, p.7), se popularizaram a partir de 1998, quando a utilização da Internet tornou-se lugar comum. "O avanço das empresas '.com' estava no seu auge, levando a uma quantidade extrema de atividades de desenvolvimento web". Logo, sistemas computacionais e interfaces acessíveis passaram a ser consideradas tecnologias em rápida disseminação. Como caracteriza Castells (2003, p.258), "os produtores de tecnologia da Internet foram fundamentalmente seus usuários". Não obstante, segundo Winckler e Pimenta (2002, p.86) "observa-se que tal popularidade não implica necessariamente em usuários satisfeitos", aumentando assim, a atenção para um fator conhecido como usabilidade, a qual permite

que pessoas com baixo grau de instrução possam ter empregos significativos, podemos conectar idosos com a comunidade, podemos fornecer as mesmas informações e serviços a usuários portadores de deficiências físicas que fornecemos a todas as outras pessoas, podemos permitir que todo mundo utilize computadores de uma maneira mais produtiva e reduzir seus sentimentos de frustração e impotência. A maior recompensa é que essas melhorias na qualidade de vida não vêm à custa dos seus lucros. Pelo contrário, a usabilidade beneficia os negócios e beneficia a humanidade. (NIELSEN; LORANGER, 2007, p.24).

Nesse sentido, Nielsen (apud MULLET; SANO, 2006, p.IX) explica que sistemas de usabilidade são compostos por diversos atributos de qualidade, dentre os quais se destacam: (i) a facilidade de aprendizado; (ii) a eficiência na utilização; (iii) a memorização; (iv) a menor proporção a erros do usuário e, (v) a satisfação subjetiva. Os fatores que se relacionam com a garantia de tais atributos estão relacionados com um projeto de interface que faça uso dos princípios da comunicação visual, bem como o emprego dos métodos sistemáticos da engenharia de usabilidade.

Neste contexto, este artigo apresenta o projeto de um Ambiente Virtual de Aprendizagem centrado no usuário - o Flow. Este tipo de projeto coloca o usuário de determinado produto ou serviço no centro de discussão, ou seja, o desenvolvimento se dá através das características e necessidades do usuário, demonstrando as informações que ele deseja encontrar. Uma abordagem como essa se relaciona diretamente com a questão da usabilidade. Portanto, o problema de pesquisa que cerca este trabalho consiste em "como atingir os princípios de usabilidade em ambientes virtuais de aprendizagem (AVAs)?”.

Escolheu-se trabalhar com interfaces de ambientes virtuais de aprendizagem, uma vez que a área de Educação a Distância $(E a D)$ é a modalidade de ensino que mais utiliza tecnologias de informação e comunicação para apoiar os processos de ensino e de aprendizagem, as quais serão responsáveis por mediar o processo de comunicação que ocorrerá entre professor-professor, professoraluno e aluno-aluno. Assim, o artigo apresenta, na seção 2, aspectos que envolvem o projeto do ambiente virtual proposto. $\mathrm{Na}$ seção 3 são destacadas algumas diretrizes para o projeto centrado no usuário de AVAs. Já a seção 4, apresenta as considerações finais.

\section{Projeto do Ambiente Virtual Proposto - Flow}

O AVA Flow foi projetado pensando-se na diferença de funcionamento existente em diferentes navegadores de Internet (Firefox, Internet Explorer, Google Chrome, Safari, Opera, etc) e dispositivos móveis como celulares e 
tablets, os quais, se configuram automaticamente, independente da resolução utilizada. Desta forma, se presume que esses dispositivos sejam de fácil utilização já que a curva de aprendizagem esperada deve ser baixa. A interface do Flow foi elaborada visando o lado intuitivo, de maneira clara e limpa, procurando seguir os padrões descritos nos princípios de usabilidade (NIELSEN, 2005) integrados diretamente com os princípios universais de projeto (LIDWELL; HOLDEN; BUTLER, 2010).

Pode-se dizer que diversos autores do Desenho Industrial já desenvolveram e categorizaram métodos para projetação, bem como suas etapas. Porém, no projeto do Flow, a realização do desenho de comunicação para mídia eletrônica, caracterizado como o desenho da interface de um AVA, fez uso da metodologia idealizada por Meurer e Szabluk (2009, p.1) conhecida como Projeto E, a qual se baseia em "conceitos, definições, métodos e processos de autores consagrados em design, estruturados de acordo com as etapas sugeridas por Garrett (2003)". O Projeto E consiste em "uma metodologia projetual com aplicação prática em projetos profissionais e acadêmicos" da área de Desenho Industrial que surgiu para sanar a necessidade de definição sobre um procedimento de projeto que pudesse ser utilizado em interfaces gráficas para diferentes tipos de produtos interativos dígito-virtuais.

A interface do AVA Flow apresenta uma coerência formal e morfológica nos aspectos gráfico-visuais, além de mostrar todas as informações pertinentes em poucos cliques, pois seus usuários necessitam de respostas rápidas, muitas vezes por serem experientes e dinâmicos, facilitando o uso para os usuários novatos. Suas cores são modernas e remetem à tecnologia sem perder a seriedade, delimitando assim, as diferentes funções com clareza, enquanto que os comandos e as ações fazem uso de ícones com uma descrição de sua função quando o mouse para em cima de qualquer um desses atributos, facilitando a assimilação da tarefa prestes a ser executada. A Figura 1 apresenta a estrutura de funcionalidades e ferramentas do Flow. 


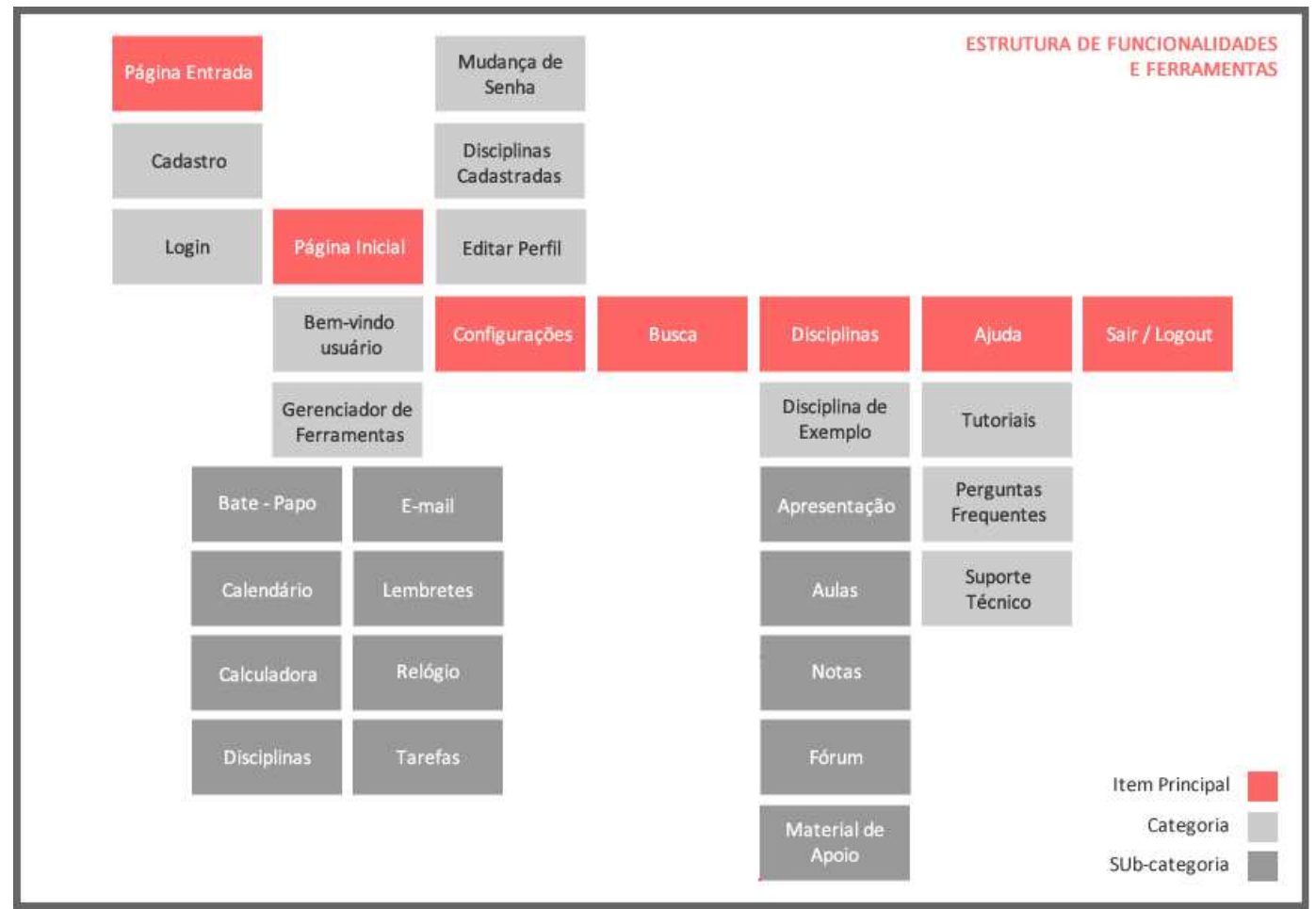

Figura 1 - Estrutura de funcionalidades e ferramentas do Flow

A seguir, encontram-se os princípios universais de projeto que foram levados em consideração para a composição dessa interface:

- Acessibilidade: implementação de ferramentas como: teclado virtual, comandos de voz (leitores de tela) e função para aumentar ou diminuir as fontes;

- Affordance: atributos que indicam seus recursos claramente com desenhos e textos coerentes, tornando a interface intuitiva;

- Camadas: conteúdo separado e relacionado por categorias (ordem);

- Carga de Desempenho: tarefas dinâmicas e fáceis de serem identificadas e assimiladas pelos usuários;

- Condescendência: interface interativa, solicita confirmação do usuário quanto a realização de tarefas consideradas "de risco";

- Confirmação: a interface mantém o usuário informado e pede sua autorização quanto à realização de quaisquer tarefas;

- Congelamento/Fuga/Luta/Entrega: utilização de atalhos para diminuir a sensação de estresse do usuário;

- Consistência: padrão visual coerente, a fim de não confundir os usuários;

- Controle: dinâmica de interação simples para que usuários experientes e novatos tenham controle do processo de interação;

- Custo/Benefício: se o usuário interagir efetivamente com a interface ela trará custo/benefício positivo;

- Efeito Estética/Usabilidade: padrão visual que busque aliar estética para que seja atrativo ao usuário e, ao mesmo tempo, seja fácil de ser compreendido e utilizado;

- Efeitos de Interferência: utilização de elementos que auxiliam a carga de desempenho do cérebro, não oferecendo muitas informações; 
- Erros: interface estruturada, buscando minimizar erros. Quando estes ocorrerem, devem ser claramente apresentados ao usuário sugerindo uma solução a ser seguida;

- Facilidade de Leitura: textos, títulos, rótulos e links devem conter mensagens adequadas com uma sintaxe formal bem elaborada, facilitando a compreensão e assimilação dos conteúdos apresentados na interface;

- Feedback: proporcionar ao usuário retorno das ações executadas através de mensagens e elementos visíveis;

- Hierarquia: apresentar as informações de forma ordenada e hierárquica;

- Imersão: os conteúdos devem ser apresentados de forma que não sobrecarreguem os sistemas perceptuais e cognitivos dos usuários, quando expostos a uma mesma atividade, durante um longo período de tempo;

- Lei de Fitts: botões e demais elementos de controle devem possuir um tamanho razóavel, a fim de aumentar a probabilidade de serem vistos, ajudando o usuário a realizar as ações dentro da interface;

- Lei de Hick: simplificar as opções, a fim de fazer com que o usuário tome a decisão certa na realização de tarefas;

- Linha de Desejo: manter um padrão coerente para que os usuários encontrem de forma lógica o que procuram;

- Mapeamento: a interface deve fazer uso de padrões já estipulados para que o mapeamento seja eficaz;

- Mimetismo: os elementos da interface devem sugerir aspectos superficiais de algum objeto semelhante, se ele existir. Porém, não de maneira exageradamente falsa, pois os aspectos relevantes são a função do objeto e não a sua aparência;

- Modelo Mental: oferecer ao usuário um modelo de interação simples, de modo que a interface se torne fácil de usar;

- Modelos Conceituais: oferecer funções semelhantes às que existem fora do mundo virtual;

- Nudge: saber esconder e limitar informações, bem como oferecer uma nova forma de interagir, quando necessário;

- Personas: usar personagens fictícios, a fim de contextualizar informações oferecidas na interface;

- Pirâmide Invertida: pode ser utilizada em textos, a fim de interagir mais com os usuários;

- Ponto de Entrada: evitar erros, fornecendo mensagens claras, desde o começo da interação para que o usuário goste desde a primeira impressão da interface em que está acessando;

- Reconhecimento sobre Lembrança: proporcionar ao usuário opções visíveis, como atalhos, para simplificar a busca durante a interação;

- Regra 80/20: procurar as ferramentas mais utilizadas pelos usuários, buscando seu constante aprimoramento;

- Relação Sinal/Ruído: a interface deve ser limpa e com o menor número possível de informações irrelevantes;

- Representação Icônica: a utilização de ícones auxilia na carga de desempenho da interface, porém estes devem ser claramente simples e fáceis de serem interpretados pelos usuários;

- Restrições: a interface deve oferecer opções que limitem as opções aos usuários, minimizando a probabilidade de erros; 
- Revelação Progressiva: as informações complexas devem ser apresentadas aos poucos, de modo que não sobrecarregue o usuário de informações, principalmente os novatos;

- Visibilidade: elementos, textos e demais informações relevantes devem estar visíveis dentro da interface, facilitando a realização das tarefas e evitando erros;

- Wayfinding: a interface deve oferecer uma espécie de "mapa de localização" da interface, que mostre os conteúdos disponíveis, bem como a sua localização, ajudando o usuário a encontrar informações e links.

As Figuras 2 e 3 apresentam algumas telas desenhadas para o Modelo Funcional Navegável da interface do AVA, que passaram por uma avaliação heurística de profissionais e pesquisadores em Interação Humano-Computador, Ergonomia Cognitiva e Desenho Industrial.

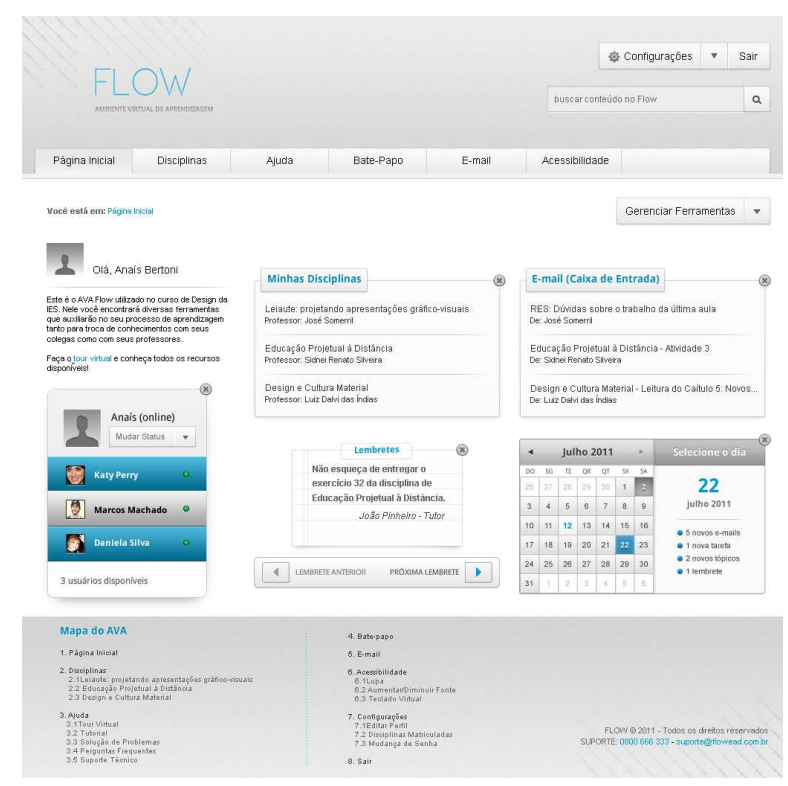

Figura 2: Página Inicial 


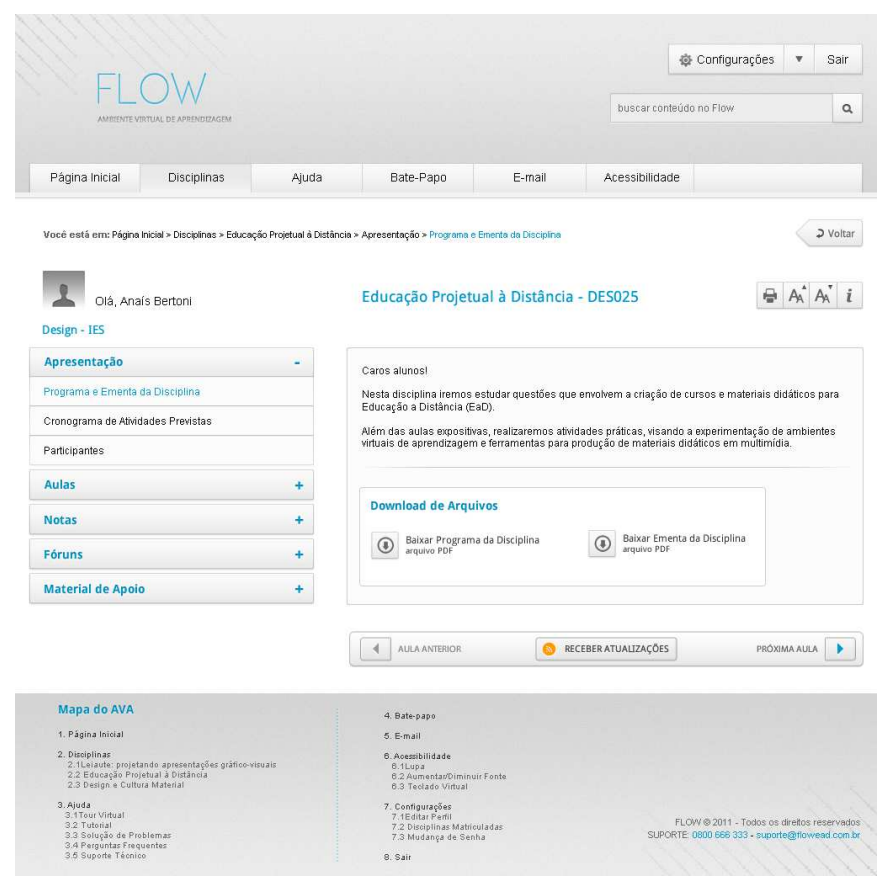

Figura 3 - Página de disciplinas

\section{Diretrizes para o Projeto Centrado no Usuário de AVAs}

Diretriz pode ser considerada um conjunto de instruções ou indicações para se levar a termo um negócio ou uma empresa; ou ainda, uma linha a qual se traça um plano de qualquer caminho. Nesse sentido, as diretrizes que serão apresentadas poderão servir como base de fundamentação na projetação de interfaces gráficas para AVAs, de modo a auxiliar desenhadores de produtos dígito-virtuais em situações comunicacionais para a área da Educação.

Para poder se chegar a diretrizes para o projeto de interfaces gráficas para AVAs, foram estudados conceitos relativos ao Desenho Industrial, à comunicação visual, à interação humano-computador, à usabilidade e à educação a distância. Após essas fundamentações foi elaborado um aprofundamento na conceituação de interfaces gráficas com o usuário, para ser possível, então, realizar um estudo sobre o desenvolvimento de projetos de produtos dígito-virtuais e princípios universais de projeto que poderiam ser aplicados no desenho de AVAs.

O desenho da interface do AVA Flow sofreu uma avaliação heurística, que consiste em uma verificação de usabilidade, realizada por profissionais de Desenho Industrial, Informática, Ergonomia Cognitiva e Interação HumanoComputador, sendo possível observar, dessa forma, que sem os requisitos listados na sequência, o desenvolvimento desse tipo de interface ficaria complexo. Por essa razão, acredita-se que as diretrizes servirão como referência para os desenhadores de produtos dígito-virtuais, pois apesar de ser uma pequena listagem de tópicos relevantes, acredita-se que se esses profissionais aliarem esses requisitos aos seus conhecimentos em desenhos, poderá haver uma unidade formal e coerente nos projetos centrados no usuário. Foram definidas, então, dez diretrizes:

a) Construção de conhecimentos: a fase que antecede o desenvolvimento de um produto consiste basicamente na fundamentação dos assuntos que o envolvem, ou seja, deve-se buscar saber o seu contexto, como ele funciona e qual é o seu propósito. Nada é diferente no caso de produtos dígito-virtuais, os 
quais envolvem a interação do usuário e, consequentemente, trabalharão com o processo cognitivo de cada um deles. A interface gráfica deverá respeitar usuários novatos, intermediários e experientes. Assim, deve-se centrar o projeto em torno deles;

b) Contextualizando o projeto: a fim de facilitar o processo de construção da interface do AVA deve-se realizar uma contextualização do projeto. Essa contextualização envolve o reconhecimento de necessidades, a textualização dos conceitos que estão sendo trabalhados por meio de, conforme Gomes (2011, p.123-142), denotações (definições extraídas de dicionários, enciclopédias e almanaques), conotações (definições extraídas de bibliografias, revistas, artigos e outros materiais) e taxonomias (hierarquização de fatores que interferem no projeto). É possível, também, elaborar um posicionamento de cenários em relação aos atores envolvidos no contexto de ambientes virtuais de aprendizagem, ou seja, identificar os cenários relacionados aos usuários (alunos e professores), às instituições de ensino e aos desenhadores, o que proporcionará uma visão ampliada dos requisitos e das necessidades que aparecerão ao longo do processo, além de sanar dúvidas, que muitas vezes, são responsáveis pelo atraso no cronograma;

c) A metodologia projetual: além de organizar o planejamento e orientar a criatividade do desenhador, a metodologia projetual será o roteiro que deverá ser seguido para o início da interface gráfica. Pode-se dizer, de uma maneira geral, que alguns métodos irão dar maior ênfase a uma determinada etapa do que a outra, além de alterar a ordem e a nomenclatura de algumas etapas, mas mesmo assim, a maioria deles apresenta uma estrutura similar, como: (i) identificação e definição de uma necessidade e/ou oportunidade de projeto; (ii) coleta de dados (informações teóricas, de mercado, etc.); (iii) geração de alternativas; (iv) seleção de alternativas e, (v) desenvolvimento do produto. Nesse sentido, no caso de desenhos dígito-virtuais, por exemplo, sugere-se a utilização da Metodologia Projetual E (ou Projeto E), desenvolvida por Meurer e Szabluk (2009), pelo fato de englobar métodos e técnicas clássicas e, ao mesmo tempo, outras específicas para o desenvolvimento de desenhos de interfaces gráficas;

d) Lista de verificação do projeto: é importante listar todos os aspectos, bem como elementos que fazem parte do projeto. Nessa lista é necessário definir a linguagem que será utilizada em relação ao seu público, as ferramentas de informação e comunicação que estarão disponíveis no projeto, a forma de interação que será trabalhada para que se realize o primeiro esboço da arquitetura de informação que irá compor o AVA. Esta lista deve ser verificada antes e depois da execução do projeto;

e) Planejamento: a organização das etapas e dos prazos é essencial para o sucesso do projeto, especialmente quando a necessidade do cliente está em questão. Por isso, é indispensável a criação de um cronograma pessoal, pois ele será responsável por organizar e documentar todas as fases do desenvolvimento da interface gráfica. Tentar seguir criteriosamente os prazos que foram estipulados é essencial, porém, sugere-se planejar um pequeno intervalo de tempo a mais para cada fase do projeto, se por acaso, algum imprevisto vier a ocorrer durante a execução do mesmo;

f) Arquitetura de informação: a arquitetura de informação é o esqueleto de informação que compõe a interface. Por meio desta arquitetura é que há a 
definição dos conteúdos através de links, botões, etc., formando a rota que poderá ser traçada dentro da interface. O ideal é levantar todos os dados e requisitos necessários que um AVA exige e relacionar esses dados para poder organizar todas as informações. Esse processo pode ser realizado por meio de card sorting, que segundo Agner (2009, p.133), consiste em uma técnica de categorização de cartões baseada nos modelos mentais dos usuários. Se o desenhador estiver trabalhando em equipe, sugere-se que esta técnica seja realizada por todos os envolvidos no projeto, de modo a desenhar, de uma forma mais próxima à realidade do usuário e sua forma de interação dentro da interface;

g) Classificação dos princípios universais de projeto: esses princípios auxiliarão no desenvolvimento da linguagem visual que será adotada no projeto de interface gráfica do AVA. Além de se referirem a diferentes normas, considerações e tendências que fazem parte das disciplinas de Desenho Industrial, esses princípios atuarão na resolução de problemas de comunicação. Isto é, a utilização desses elementos contribuirá para a obtenção de objetivos, sendo um deles, a usabilidade. Os princípios universais de projeto relacionados com a usabilidade podem ser trabalhados juntamente com outros (de percepção, de compreensão, de persuasão e de decisão) a fim de garantir que o usuário realize determinada ação dentro da interface, compreenda o que está sendo solicitado, busque informações e crie uma experiência positiva com o AVA, independente do dispositivo (computador, telefone celular, tablet) que ele esteja utilizando para efetivar o processo de interação;

h) Classificação dos 10 princípios de usabilidade: é importante dominar os 10 princípios de usabilidade propostos por Nielsen (2005), além das questões a que eles se referem. Os princípios de usabilidade auxiliarão no desenvolvimento do desenho, pois poderão atuar juntamente com os princípios universais de projeto, de forma a orientar os elementos que poderão ser inseridos e organizados no layout da interface gráfica, regidos pela malha diagramacional utilizada e definida previamente pelo desenhador. Outro motivo importante relacionado com os princípios de usabilidade está ligado ao fato de prevenção de erros de projeto, pois uma vez que o desenhador os domine, ele conseguirá relacionar mais facilmente os elementos que devem ser inseridos na interface em relação àquilo que foi estipulado na arquitetura de informação, além da maneira que acontecerá a navegação e o processo de interação do usuário com a interface, facilitando a forma de projetar;

i) Verificação do desenho do projeto de interface gráfica: é importante que o desenho da GUI passe por uma verificação, antes de ser iniciada a implementação do AVA em uma linguagem de programação, que será responsável por fazer que a interface transforme-se em um sistema interativo. Diversos autores apresentam técnicas de verificação de interface, em que se levam em consideração os requisitos de usabilidade. Dentre elas, Santa Rosa e Moraes (2008) ressaltam: técnica grupo de foco; instrumentos para arquitetura de informação; avaliação heurística; card sorting; avaliação cooperativa; teste de usabilidade; questionário de satisfação do usuário e técnicas de prototipagem. Uma vez que a interface gráfica do AVA seja testada por um ou mais desses métodos de verificação, faz-se necessário a classificação dos erros para identificar a natureza deles, por exemplo, erros de desenho ou erros de 
usabilidade. Uma vez feita essas considerações, o desenhador deve iniciar a etapa de correção da interface;

j) Correções no desenho da interface gráfica: devem-se corrigir todos os problemas e/ou falhas encontrados na etapa de verificação da interface, pois mesmo aqueles que possuam baixo grau de relevância, de uma forma ou de outra, poderão influenciar no processo de cognição dos usuários. Sugere-se que, uma vez que a interface seja corrigida, ela passe por uma nova avaliação no desenho, a fim de garantir que o projeto trará ao desenhador o retorno de investimento em usabilidade antes do AVA ser implementado em uma linguagem de programação pelos profissionais de informática.

As diretrizes apresentadas podem ser seguidas por desenhadores para o projeto de interfaces gráficas de AVAs, pois apresentam aspectos que esses profissionais devem prestar atenção durante a execução desse tipo de produto dígito-virtual.

\section{Considerações Finais}

Tendo em vista os aspectos observados durante o desenvolvimento deste trabalho, percebe-se a importância da usabilidade no projeto de interfaces gráficas, já que ela não só é considerada um atributo de qualidade importante, como necessário no que diz respeito aos produtos dígito-virtuais, principalmente aqueles de caráter educacional. Projetar um AVA é um desafio, mesmo para os desenhadores mais experientes, já que se constitui em um tipo de interface complexa, pelo fato de envolver diversos tipos de recursos, tanto de tecnologias como de conteúdos. O projeto educacional exigirá uma carga cognitiva muito elevada do desenhador e uma rotina de trabalho amplamente focada no cronograma e no funcionamento das ferramentas que serão implementas na interface. Tais fatores serão determinantes durante a experiência do usuário.

O resultado da avaliação heurística realizada no AVA Flow não apresentou problemas graves de usabilidade, o que permitiu que as correções fossem elaboradas sem maiores dificuldades. O domínio dos princípios de usabilidade por parte do desenhador se mostrou um fator essencial para a escolha dos princípios universais de projeto, pois auxiliaram na escolha dos mesmos, identificando aquilo que é permitido e coerente no projeto de uma situação comunicacional para a área da Educação.

As ideias apresentadas e os estudos realizados permitiram perceber que fica evidente a necessidade de um aprofundamento das questões de usabilidade e dos princípios universais de projeto para estudos futuros. A aplicação desses conceitos em situações comunicacionais para Educação é importante, pois se observa que ainda há muito mais o que se trabalhar, principalmente, no que diz respeito às ferramentas de interação dos usuários, como wikis, fóruns, ferramentas de edição de textos e exercícios colaborativos, que venham a fazer parte de um AVA. Tais ferramentas podem estimular a interação em AVAs e potencializar os processos de ensino e de aprendizagem, dependendo da forma como elas podem vir a serem projetadas, visto que há uma necessidade de aprimoramento das ferramentas utilizadas nos cursos a distância, seja devido ao aumento da oferta e da procura por cursos nessa modalidade. Os usuários de AVAs necessitam de Tecnologias de Informação e Comunicação que contribuam de maneira eficaz e 
eficiente nos processos de ensino e de aprendizagem, e o desenhador de interfaces gráficas pode ser um importante personagem nesse contexto.

\section{Referências}

AGNER, Luiz. Ergodesign e arquitetura de informação: trabalhando com o usuário. 2. ed. Rio de Janeiro: Quartet, 2009.

ARANTES, Priscila. Arte e mídia: perspectivas da estética digital. São Paulo: Ed. Senac São Paulo, 2005.

CASTELLS, Manuel. Internet e sociedade em rede. In: MORAES, Dênis de (org.). Por uma outra comunicação: mídia, mundialização cultural e poder. Rio de Janeiro: Record, 2003, p.255-287.

GARRETT, Jesse James. The elements of user experience: user-centered design for the web. New York: AIGA, 2003.

GOMES, Luiz Antônio Vidal de Negreiros. Criatividade e design: um livro de desenho industrial para projeto de produto. Porto Alegre: sCHDs, 2011.

LÉVY, Pierre. As tecnologias da inteligência: o futuro do pensamento na era da informática. Rio de Janeiro: Ed. 34, 1993.

LIDWELL, William; HOLDEN, Kritina; BUTLER, Jill. Princípios Universais do design. Trad. Francisco Araújo da Costa. Porto Alegre: Bookman, 2010.

MEURER, Heli; SZABLUK, Daniela. Projeto E: aspectos metodológicos para o desenvolvimento de projetos dígito-virtuais. In: 9 ERGODESIGN | 9 USIHC, 2009, Curitiba. Anais do 9 ERGODESIGN | 9 USIHC, 2009.

MULLET, Kevin; SANO, Darrell. Designing visual interfaces: communication oriented techniques. California: Sunsoft, 2006.

NIELSEN, Jakob; LORANGER, Hoa. Usabilidade na web: projetando websites com qualidade. Rio de Janeiro: Elsevier, 2007.

NIELSEN, Jakob. Ten usability heuristics. Useit.com, jan. 2005. Disponível em: <http://www.useit.com/ papers/heuristic/heuristic_list.html>. Acesso em: 21 abr. 2011.

SANTA ROSA, José Guilherme; MORAES, Anamaria de. Avaliação e projeto no design de interfaces. Rio de Janeiro: 2AB, 2008.

WINCKLER, Marco; PIMENTA, Marcelo Soares. Avaliação de Usabilidade Web. In: NEDEL, Luciana Porcher (Org.). X Escola de informática da SBC-Sul. Porto Alegre: Instituto de Informática - UFRGS, 2002, p.85-137. 